\title{
Neutron Scattering from Solutions: The Hydration of Lanthanide and Actinide Ions CONF-8509147--3 \\ DE85 017711
}

\author{
R. L. Hahn, A. H. Narten, and B. K. Annis \\ Oak Ridge Nâtional Laboratory, Oak Ridge, TN 37830, USA
}

\section{Summary}

The neutron scattering difference method is described and applied to investigations of the aqua rare-earth ions, $\mathrm{Nd}^{3+}$ and $\mathrm{Dy}^{3+}$. Metal-water distances and hydration numbers have been unambiguously determined for these ions' inner coordination spheres. Tine values of the hydration number, $n$, of $8.5 \pm 0.2$ for $\mathrm{Nd}^{3+}$ and $7.4 \pm 0.5$ for $\mathrm{Dy}^{3+}$ : directly support the claim of Spedding et al. that $n$ decreases by one unit across the lanthanide series. The possible application of this method to actinide ions in solution is also discussed.

\section{Introduction}

The study of the properties of hydrated ions is of fundamental chemical significance, since many of the icn's interactions in aqueous solution depend upor. these properties; e.g., complexation of the ion occurs when a water molecule in the ion's inner coordination shell is replaced by the complexing iigand. Many different methods have been used to study hydrated ions [1]. Some of these methods measure bulk properties of solutions, such as enthalpies and entropies of solution, or ionic mobilities, while other methods probe the local environment around the rydrated ion. The latter "microscopic" methods include 
optical spectroscopy, n.m.r., e.?.r., x-ray diffraction and neutron scattering $[1]$.

In this paper, we discuss neutron scattering, and how it applies to investigations of $L n$ and $A n$ aqua ions. Because the neutron scattering data from a solution contain contributions from a 71 of the atom-pair interactions therein, analysis of the scattering pattern of a salt dissolved in water is complicated and not unique. A significant simplification of the data can be effectuated by the difference method, which was first described and exploited by Enderby and coworkers [2].

\section{Principles of the Neutron Scattering Difference Method}

The usual scattering experiment invoives measuring the distribution of scattered neutrons of wavelength, $\lambda$, as a function of angle, $2 \theta$, or of transferred momentum, $k=(4 \pi / \lambda) \sin \theta$. The chemical or structural information from the scattering distribution can be expressed as

$$
\rho \sum_{\alpha \beta} c_{\alpha} c_{B} f_{\alpha} f_{B} \hat{h}_{\alpha \beta}(k) \text {. }
$$

Here $\alpha$ and $B$ represent different species in the solution, $\rho$ is the number density, $c_{\alpha}$ is the atom fraction and $f_{\alpha}$, the coherent scattering length, of atom $\alpha$.

The rtructure function is

$$
\hat{h}_{\alpha \beta}(k)=4 \pi \int_{0}^{\infty} r^{2} h_{\alpha \beta}(r) j_{0}(k r) d r
$$

with $j_{0}(k r)=\sin \mathrm{kr} / \mathrm{kr}$. The desired atom-pair distribution function is

$$
g_{\alpha \beta}(r)=h_{\alpha \beta}(r)+I \text {, }
$$

For a sample of an ionizable salt, $M X_{\Pi}$, dissolved in water, the neutron scattering pattern is the surn of contributions from ten atom-pair distribucions. Enderby et al. [2] have simplified the data analysis for such a sample by using isotopic substitution. Because 
neutron scattering is a nuclear phenomenon, the scattering length of element $M$ depends on its isotopic composition. If we prepare (at least two) solutions of $M X_{n}$ that are identical chemically and differ only in the isotopic compositions, $i$ and $j$, of element $M$, the neutron scatiering patterns of the solutions will differ only becasse of the differences between $f_{i}$ and $f_{j}$. If we then take the difference of the (two) scattering data sets, only terms involving element $M$ will survive; the differences will be identically zero for the other species in the solution. Instead of the original scattering pattern involving ten terms, the difference pattern will contain only four terms and will be amenable to analysis.

\section{Experimental Procedures}

We have applied the difference method to the study of the trivalent lanthanide aqua ions, $\mathrm{Nd}^{3+}$ and $\mathrm{Dy}^{3+}$. Details of these experiments are discussed in Refs. $[3,4]$.

Matched aqueous solutions, but with differing metal-ion isotopes, were prepared of $\mathrm{NdCl}_{3}$ and of $\mathrm{DyCl}_{3} \cdot \mathrm{D}_{2} \mathrm{O}$ was used as the solvent because light hydrogen has a large incoherent scattering cross-section. Concentrations of the solutions were first measured by EDTA titrations, and the firal solutions were then made identical by dilution by weight. The compositions of the solutions are listed in Table 1.

The neutron experiments were carried out at the High Flux Isotope Reactor with a beam of $0.889-8$ neutrons. A linear position-sensitive proportional counter (PSPC) was used to collect the data. The zounter covered an angular range of $42^{\circ}$. Scattered intensities were recorded over an angular range of $7^{\circ}<2 \theta<133^{\circ}$. The average number of 
accumulated counts was about $3 \times 10^{6}$ per degree of scattering angle. The data were stored in bins of constant $\Delta k=0.1 \AA^{-1}$.

The measured intensities were corrected for background, and the absolute scattering cross-section scale was determined with a solid vanadium cylinder having the same dimensions as the samples. The data were then corrected for multiple scattering and absorpticn in the sample, and absorption and scattering by the sample container.

4. Results and Discussion for Lanthanides

The differential scattering cross-sections for the four different $\mathrm{NdCl}_{3}$ solutions are shown in $\mathrm{Fig}$. 1. To remove the contributions to the scattering cross-sections from interactions that do not involve the $\mathrm{Nd}$ ion, we subtracted the data for the ${ }^{144} \mathrm{NdCl}_{3}$ solution from those of the other three solutions. The resulting differences may be written as $\Delta^{\circ}(k)=\Delta(k)+\Delta_{S}(k)$, with $\Delta_{S}(k)$ the self-scattering from unccrrelated atoms. The three difference curves $\Delta(k)$ thus derived from the four data sets are shown in Fig. 2.

The difference functions for the solutions with the nuclides $i={ }^{\text {nat }} \mathrm{Nd},{ }^{142} \mathrm{Nd}$, or ${ }^{146} \mathrm{Nd}$ and $j={ }^{144} \mathrm{Nd}$ may be written as

$$
\Delta_{i j}(k)=\rho\left[A_{i j} \hat{h}_{M 0}(k)+B_{i j} \hat{h}_{M D}(k)+C_{i j} \hat{h}_{M C]}(k)+D_{i j} \hat{h}_{M M}(k)\right]
$$

with

$$
\begin{aligned}
& A_{i j}=(2 c / 3)(1-4 c) f_{0}\left(f_{i}-f_{j}\right) \\
& B_{i j}=(4 c / 3)(1-4 c) f_{D}\left(f_{i}-f_{j}\right) \\
& C_{i j}=6 c^{2} f_{C l}\left(f_{i}-f_{j}\right) \\
& D_{i j}=c^{2}\left(f_{i}{ }^{2}-f_{j}{ }^{2}\right) .
\end{aligned}
$$

$f_{0}, f_{D}$, and $f_{C l}$ are the scattering factors of oxygen, deuterium, and chlorine and $i, j$ refer to the appropriate neodymium isotopes. Terms that contribute to the data in Fig. 1, but do not involve the scattering 
factor $f_{\text {Nd }}$, do not appear in Eq. 4. Combining Eq.4 with Eqs. 2 and 3 gives the desired structural information about the Nd cation. Because we have determined three difference curves instead of one, the problem is overdetermined. We have thus solved the equations by the leastsquares method, obtaining a combined structure function, $H_{N d}(k)$. The Fourier transform of $\mathrm{H}_{\mathrm{Nd}}(k)$ yields the (RDF) radial dictribution function, $G_{N d}(r)$, shown in Fig. 3. The same type of analysis for the $\mathrm{DyCl}_{3}$ solutions yields $\mathrm{G}_{\mathrm{Dy}}(r)$, Fig. 4 .

The two large peaks in each of the metal-ion RDF's, Figs. 3 and 4, can be directly and uniquely determined. In Fig. 3, e.g., the integrai

$$
N(r)=4 \pi \rho \int r^{2} G_{N d}(r) d r
$$

evaluated from the distance $R_{1}=2.20 \mathrm{~A}$, at which the first peak has zero value, to the first minimum at $R_{2}=2.80 \mathrm{~A}$, has a value $N_{1}=7.78$. The integral over the second peak from $R_{2}$ to the second minimum at $R_{3}=$ $3.55 \mathrm{~A}$ is $\mathrm{N}_{2}=17.30$. From the definition of the atom-pair distribution functions the number of $\gamma$ atoms around an $\alpha$ atom is

$$
n=4 \pi c_{\gamma} \rho \int r^{2} g_{\alpha \gamma}(i) d r .
$$

If we combine Eqs. 5 and 6 and assign the peaks at 2.5 and $3.1 \mathrm{~A}$ respectively to the $N d-O$ and $N d-D$ interactions, we find that $n_{2}=16.7$, $n_{1}=8.6$, and $n_{2} / n_{1}=1.94$. Since this ratio differs by only $3 \%$ from the expected value for $D_{2} 0$ of $n_{2} / n_{1}=2$, we conclude that our assignment of the peaks is unique. There are thus 8.6 oxygen atoms around the neodymium ion and 16.7 deuterium atoms. Taking the average $(8.6+$ $16.7 / 2) / 2$ as the best estimate of the number of water molecules around a $\mathrm{Nd}^{3+}$ ion, we obtain a hydration number, $\mathrm{n}=8.5 \pm 0.2$. The same analysis applied to the $\mathrm{DyCl}_{3}$ data gives $n=7.4 \pm 0.5$ for $\mathrm{Dy}^{3+}$. 
The neutron scattering difference method yields in a straightforward, unambiguous manner the ion-water distances and the hydration numbers for the inner coordination sphere. These values for the Nd and Dy cations are given in Table 2. The sharpness of the M-O and M-D peaks in the RDF's indicate well-defined first coordination spheres of water molecules around the lanthanide ions in solution. From the measured distances and the known geometry of water molecules in the liquid, it follows that the molecules in the first hydration sphere also have a we11-defined average orientation, with the hydrogens pointing away from the cation at a tilt angle of $\sim 20^{\circ}$, as shown in Fig. 5 .

The hydration numbers found in this work are not integers. They are average values from which local deviations certainly occur in the liquid, because the residence time of ar individual water molecule in the lydration sphere of a lanthanide ion is brief, $<10^{-9} \mathrm{~s}[1]$. The information on cationic hydration presented here is valid only for relatively concentrated solutions, $>2 \mathrm{~m}$, and the results may not be representative for dilute solutions.

Our results are supportive of the claims made by Spedding et al. [5] that, as a result of the lanthanide contraction, the hydration number of the $\mathrm{Ln}^{3+}$ ions decreases by one unit, in going from the light (e.g., Nd) to the heavy (Dy) end of the rare-earth series.

5. Extension of the Method to the Actinides

Little is known about the hydration characteristics of actinide ions. Of especial interest is the question: does the actinide contraction cause a reduction in hydration number similar to cur results 
for the lanthanides? Divid [6] has presented some arguments for such a decrease, based on radiotracer diffusion measurements.

Based on our experience with the neutron scattering difference. method, we can list the rollowing requirements for its successful utilization: (1) The element of interest must have more than one isotope. (2) The scattering lengths of its isotopes must differ by at least $10 \%$; the greater the difference, the better. (3) The minimum concentration that will give reasonable results is $20.5-1 \mathrm{~m}$.

When we apply these criteria to the actinides, we see that at present the method will work for only a few elements. Coherent scattering lengths are not known for she isotopes of many of these elements [7]. And since the sample volume is $>1 \mathrm{ml}$, hundreds of milligrams of each isotope are required; such amounts are not available for most of the isotopes above americium.

Uranium and plutonium are two elements for which the difference method should work. Their scattering lengths are [7]: $235_{U}, 0.98$; ${ }^{238} \mathrm{U}, 0.84 ;{ }^{239} \mathrm{Pu}, 0.77 ;{ }^{240} \mathrm{Pu}, 0.35 ;{ }^{242} \mathrm{Pu}, 0.81 ; \times 10^{-12} \mathrm{~cm}$. Clearly the plutonium system is more attractive, although its solution chemistry is more complicated than uranium's.

Other complicating factors in such work is that the $\mathrm{Pu}$ isotopes must be handled in an a-containment facility, with the matched solutions being prepared in a controlled atmosphere glove box to minimize the $\mathrm{H}_{2} \mathrm{O} / \mathrm{O}_{2} \mathrm{O}$ ratio. For the actual scattering experiments at the HFIR, a doubly contained system must be constructed that is not only safe, but also adds only a sma11, isotropic background to the counting data. For our experiments, the primary sample container, with $0.5 \mathrm{~mm}$ walls, is 
made of a titanium-zirconium alloy (which is also corrosion resistant). The secondary container is of vanadium metal, with $0.25 \mathrm{~mm}$ walls. Our immediate plans are to study the hydration of the $\mathrm{Pu}^{4+}$ aqua ion, using the isotopes ${ }^{240} \mathrm{Pu}$ and ${ }^{242} \mathrm{Pu}$. If this experiment is successful, we will exanine ways of stabilizing the $\mathrm{Pu}^{3+}$ ion for the length of time required for the scattering experiment (several days), to allow us to obtain a direct comparison of the hydration properties of $\mathrm{An}^{3+}$ ions with $\mathrm{Ln}^{3+}$ ions.

\section{$\underline{\text { References }}$}

1. J. Burgess, "Metal Ions in Solution" ([1lis Horwood Ltd., Chichester, 1978).

2. J. E. Enderby and G. W. Neilson in "Water: A Comprehensive Treatise", Vol. 6, F. Franks, Ed. (Plenum, New York, 1979), p. 1.

3. A. H. Narten and R. L. Hahn, J. Phys. Chem. 87 (1983) 3193.

4. B. K. Annis, R. L. Hahn, and A. H. Narten, J. Chem. Phys. 82 (1985) 2086.

5. A. Habenschuss and F. H. Spedding, J. Chem. Phys. 70 (1979) 3758.

6. F. David, Institut de Physique Nucleaire Report No. IPNO-DRE 84.01 (1984).

7. L. Koester, H. Rauch, M. Herkens, and K. Schroder, KFA Julich Report (1984); V. F. Sears, Chalk River Laboratories Report No. AECL-8490 (1984).

Research sponsored by the Division of Chemical Sciences, Office of Basic Energy Sciences, U.S. Department of Energy under contract DE-AC05-84QR21400 with Martin Marietta Energy Systems, Inc.

By acceptance of this article, the publisher or recipient acknowledges the U.S. Government's right to retain a nonexclusive, royalty-free license in and to any copyl ight covering the article. 
Table 1. Preparation of "Identical Solutions"

\begin{tabular}{|c|c|c|c|}
\hline ISOTOPE & $\%$ & $\begin{array}{l}\text { SCATTERING LENGTH } \\
\quad \mathrm{f}\left(10^{-12} \mathrm{~cm}\right)\end{array}$ & $\begin{array}{c}\text { ELEMENT FRACTION } \\
\text { c }\end{array}$ \\
\hline \multicolumn{4}{|c|}{$2.85 \mathrm{~m} \mathrm{NaCl}_{3}$ in $\mathrm{D}_{2} \mathrm{O}^{*}$} \\
\hline $\mathrm{NAT}_{\mathrm{Nd}}$ & - & 0.72 & 0.0176 \\
\hline${ }^{144} \mathrm{Nd}$ & 97.5 & 0.31 & 0.0176 \\
\hline $142 \mathrm{Nd}$ & 97.6 & 0.78 & 0.0177 \\
\hline${ }^{146} \mathrm{Nd}$ & 97.6 & 0.87 & 0.0176 \\
\hline \multicolumn{4}{|c|}{$2.38 \mathrm{~m} \mathrm{DyCl}_{3}$ in $\mathrm{D}_{2} \mathrm{O}^{*}$} \\
\hline $\mathrm{NAT}_{\text {Dy }}$ & - & 1.69 & 0.0150 \\
\hline${ }^{16]^{D y}}$ & 95.9 & 1.03 & 0.0149 \\
\hline${ }^{162}{ }^{10}$ & 92.4 & -0.14 & 0.0150 \\
\hline${ }^{164}$ Dy & 98.4 & 4.94 & 0.0149 \\
\hline
\end{tabular}


Table 2. Hydration of Rare-Earth Ions, $\mathrm{M}^{3+}$

\begin{tabular}{cccccc}
\hline $\mathrm{M}^{3+}$ & $\mathrm{r}_{M 0}(\AA)^{\mathrm{a}}$ & $\mathrm{r}_{M D}(\AA)^{\mathrm{a}}$ & $\mathrm{n}^{\mathrm{a}}$ & $\mathrm{r}_{M^{3+(\AA)}}{ }^{\mathrm{b}}$ & $\mathrm{n}_{X-\mathrm{ray}} \mathrm{c}^{\mathrm{c}}$ \\
$\mathrm{Nd}^{3+}$ & 2.48 & 3.13 & $8.5 \pm 0.2$ & 1.16 & 8.90 \\
$\mathrm{Dy}^{3+}$ & 2.37 & 3.04 & $7.4 \pm 0.5$ & 1.03 & 7.93 \\
\hline
\end{tabular}

${ }^{\mathrm{a}}$ This work

bFrom R. D. Shannon, Acta Cryst. A32 (1976) 751.

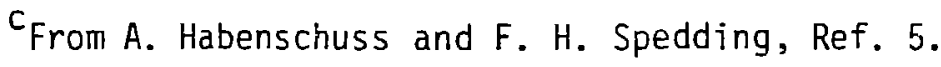


Figure Captions

Figure 1. Neutron scattering cross sections of $2.85 \mathrm{~m} \mathrm{NdCl}_{3}$ solutions, identical in every respect except for the isotopic state of the Nd ions.

Figure 2. Difference curves $\Delta(k)$ derived from the cross sections shown in Figure 1 . From top to bottom: ${ }^{142} \mathrm{Nd}-{ }^{144} \mathrm{Nd}$, nat $\mathrm{Nd}_{-}{ }^{144} \mathrm{Nd},{ }^{148} \mathrm{Nd}_{-}{ }^{144} \mathrm{Nd}$. The statistical error of an average data point is \pm 0.001 .

Figure 3. Radial distribution function for Nd interactions obtained by the Fourier transfurm of the combined structure function, $\mathrm{H}_{\mathrm{Nd}}(\mathrm{k})$.

Figure 4. Radial distribution function for Dy interactions.

Figure 5. Arrangement of a water molecule in the first hydration sphere of a cation, $\mathrm{M}^{\mathrm{nt}}$, in solution. There are 8.5 such molecules around $\mathrm{Nd}^{3+}$, and 7.4 around $\mathrm{Dy}^{3+}$. Tilt angles for both cations are $\sim 20^{\circ}$. 


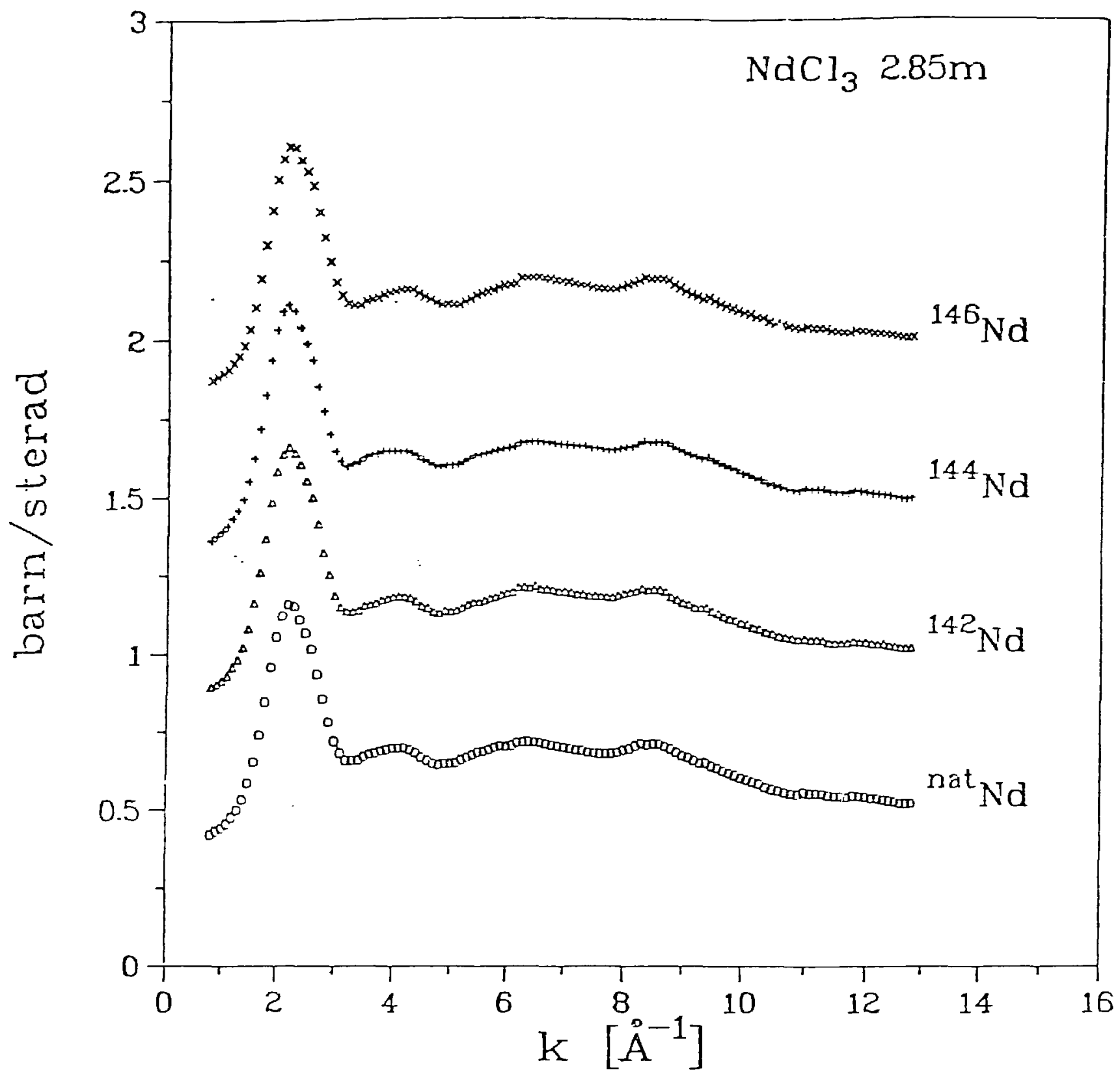

Figure 1. Neutron scattering cross-sections of $2.85 \mathrm{~m} \mathrm{NdCl}_{3}$ solutions, identical in every respect except for the isotopic state of the Nd ions. 


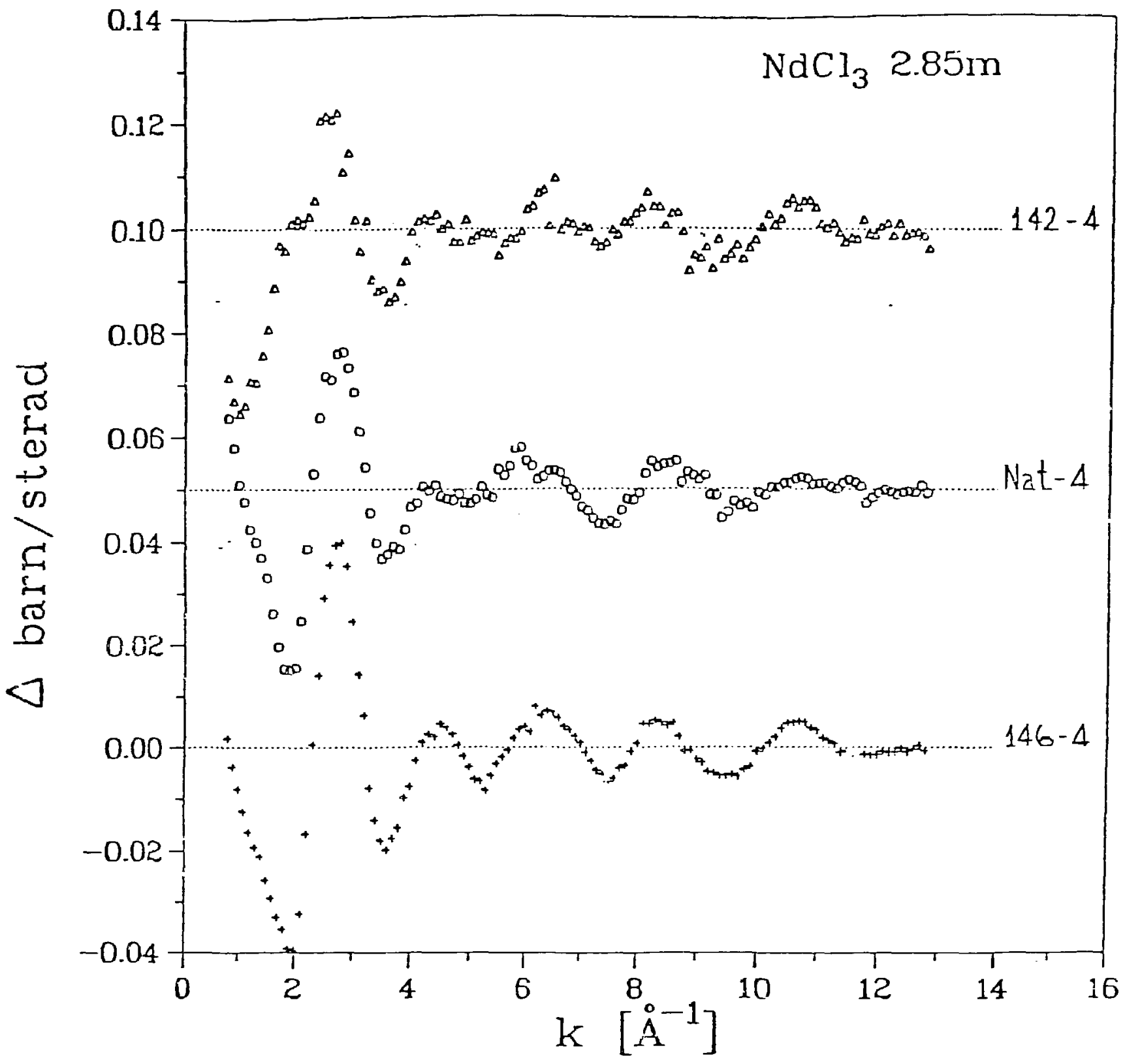

Figure 2. Difference curves $\Delta(k)$ derived from the cross sections shown in Figure 1 . From top to bottom: $142_{: \mathrm{H}_{-}}{ }^{444} \mathrm{Nd}$, nat $_{\mathrm{Nd}-}{ }^{144} \mathrm{Nd},{ }^{148} \mathrm{Nd}^{144} \mathrm{Nd}$. The statistical error of an average data point is \pm 0.001 . 


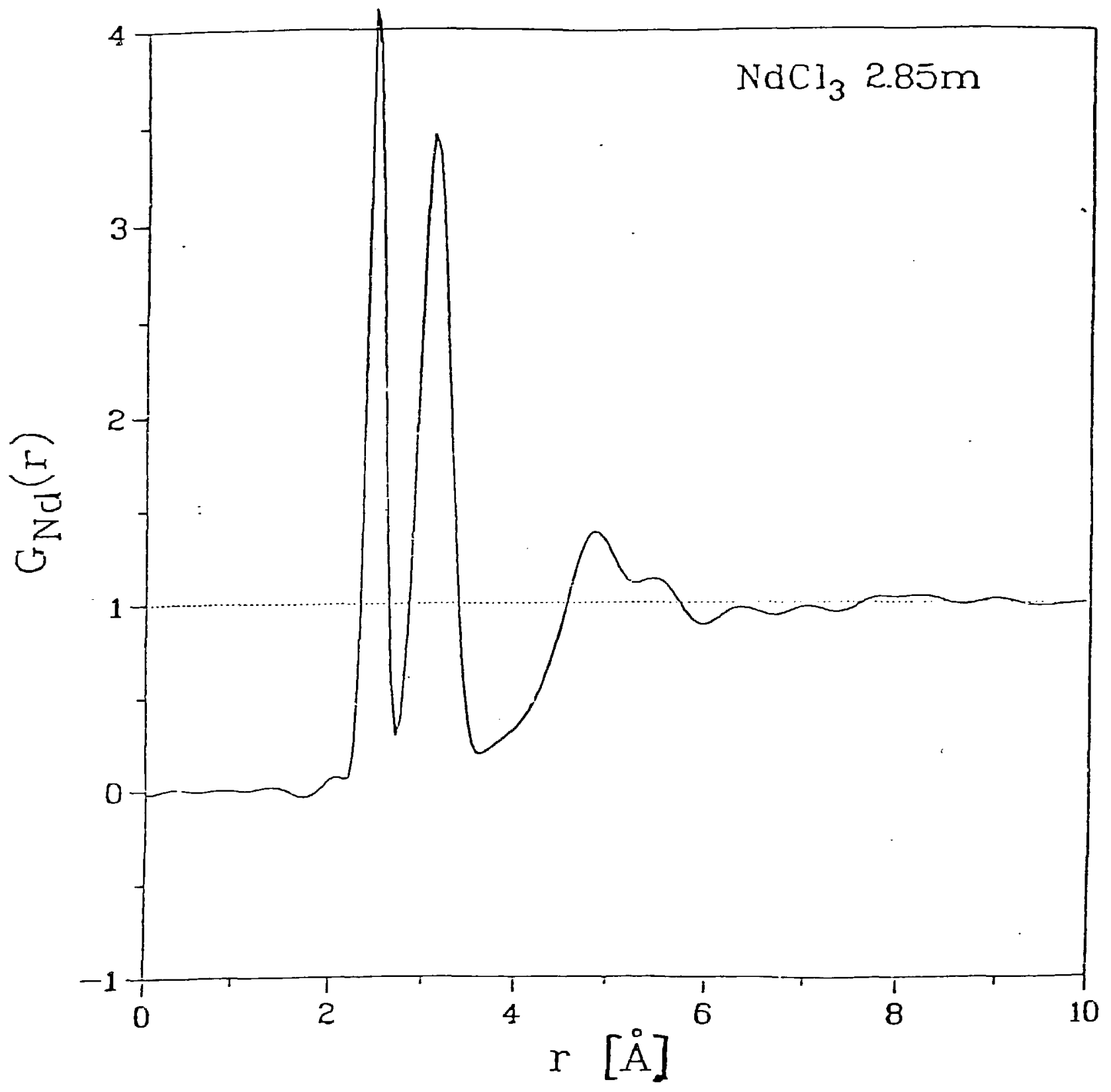

Figure 3. Radial distribution function for Nd interactions obtained by the Fourier transform of the combined structure function, $\mathrm{H}_{\mathrm{Nd}}(\mathrm{k})$. 


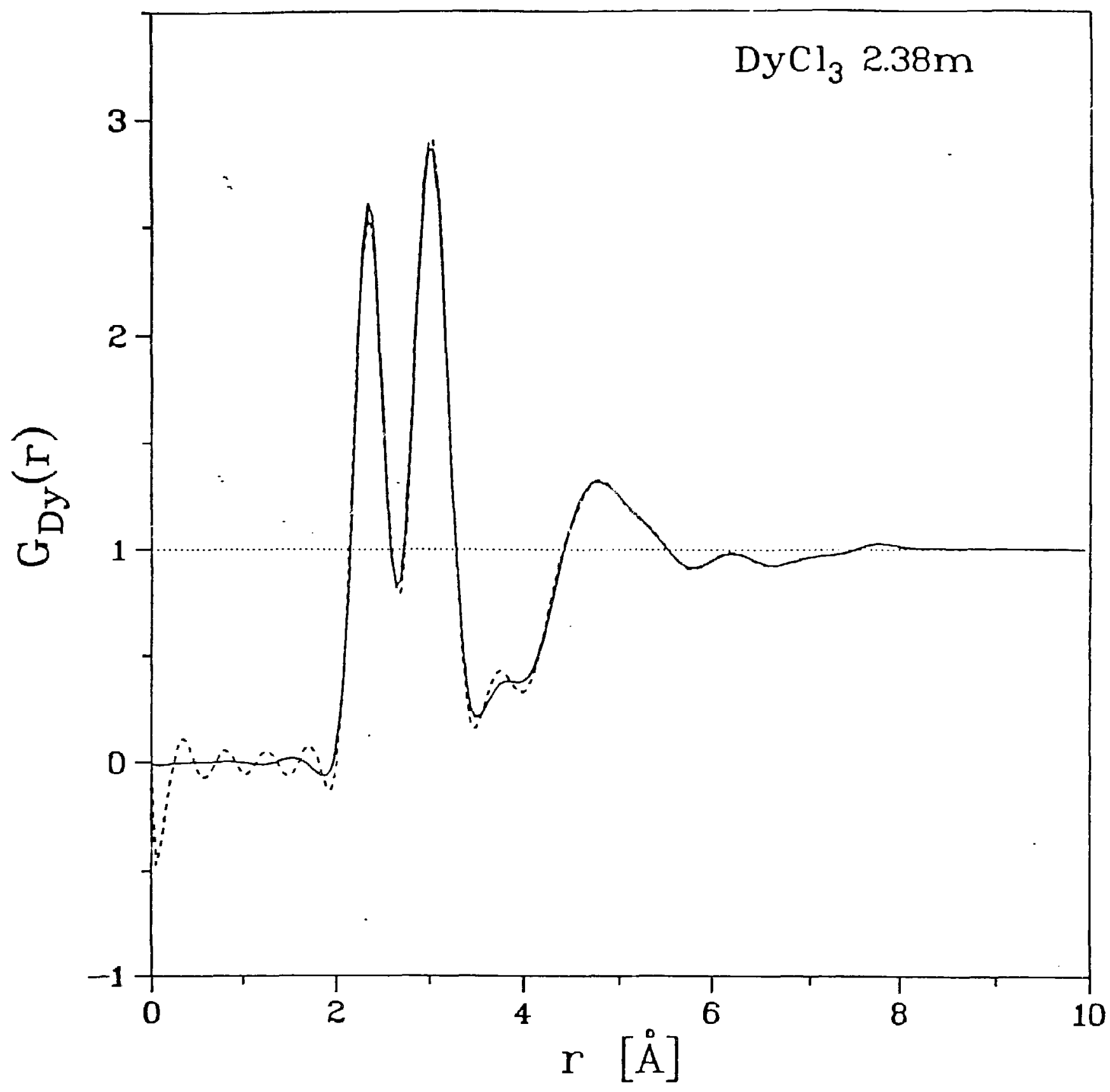

Figure 4. Radial distribution function for Dy interactions. 


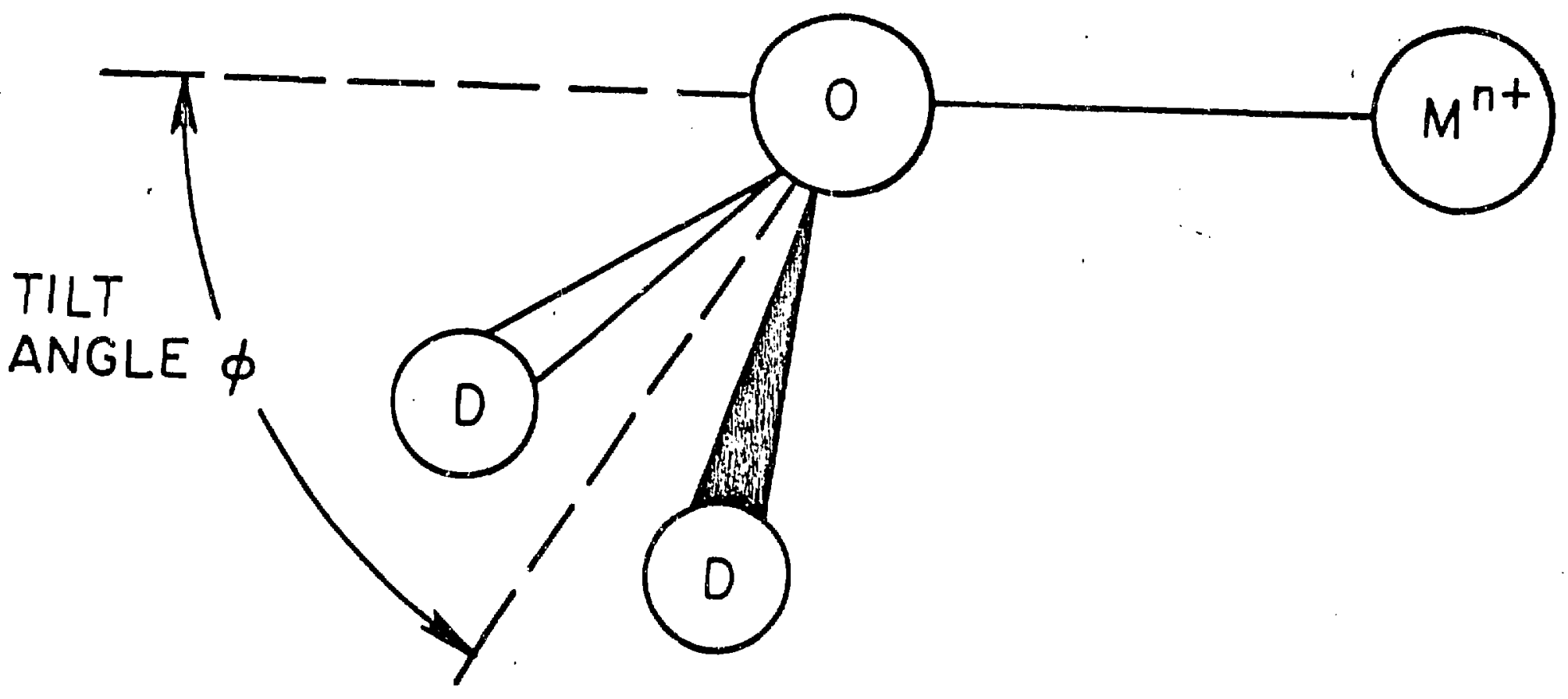

Figure 5. Arrangement of a water molecule in the first hydration sphere of a cation, $\mathrm{M}^{\mathrm{nt}}$, in solution. There are 8.5 such molecules around $\mathrm{Nd}^{3+}$, and 7.4 around $\mathrm{Dy}^{3+}$. Tilt angles for both cations are $20^{\circ}$. 